\title{
Introduction to the Special Section: Precision Teaching: Discoveries and Applications
}

\author{
Andrew J. Bulla ${ }^{1} \cdot$ Abigail Calkin $^{2}$ - Mary Sawyer ${ }^{3}$ \\ Published online: 17 August 2021 \\ (C) Association for Behavior Analysis International 2021
}

\begin{abstract}
The current article provides an introduction to the special section in Behavior Analysis in Practice focusing on precision teaching and standard celeration charting. This particular section highlights recent advancements and discoveries made using the standard celeration chart. Drs. Andrew Bulla, Mary Sawyer, and Abigail Calkin served as guest editors for the special section. This section includes articles focusing on applications to general and education settings, working with individuals with disabilities, tutorial pieces giving practitioners a step-by-step guide for implementing procedure, as well as unique applications of the standard celeration chart.
\end{abstract}

Keywords Precision teaching $\cdot$ Standard celeration chart $\cdot$ Precision measurement

Behavior Analysis in Practice has a long history of special issues and special sections devoted to a specific area within the field of behavior analysis. We are pleased to continue this tradition and offer a special section devoted to precision teaching and the standard celeration chart (SCC). We hope to bring the readers of the journal up-to-date research and information about the current state of precision teaching and the SCC. In addition, we hope to further clarify concepts and terms within the field to distinguish precision teaching from any application of the SCC.

The concepts for the standard celeration chart began with B. F. Skinner's cumulative recorder and his work with rats and pigeons. In addition, Ogden Lindsley, a graduate student of Skinner's, and his work with dogs contributed to the development of the standard celebration chart. Lindsley began using frequency to monitor human behavior, in particular, the freeoperant behavior of one of his daughters, in 1952. At that time, a Newsweek article, "Babe in a Box," described Lindsley's work with his daughter. The article stated that although about 250 infants had grown up in Air Cribs, "Kathy [Lindsley] is the first of the crowd to undertake a precise scientific

Andrew J. Bulla

abulla@georgiasouthern.edu

\footnotetext{
Georgia Southern University-Armstrong, Savannah, GA, USA

Calkin Consulting Center, Gustavus, AK, USA

Fit Learning, Atlanta, GA, USA
}

experiment" ("Babe in a Box," 1952, p. 98). The article included a photo of Kathy in her Air Crib with two rows of five lights, which were buttons his daughter could press. Lindsley monitored the button pressing of his daughter. Lindsley's daughter noted:

As [my mother, Mary Lindsley Kenny, Ogden's first wife] recalls, there was a big red button, my hand is on it in the Newsweek photo. Initially there was a two tone [sic] door chime and according to the article he later installed and substituted lights (panel of two rows of lights) would flash [sic]. So I had to press the red button. (K. L. Fandetti, personal communication email to A. B. Calkin, January 15, 2018)

Lindsley had just conducted the first behavior analytic, free operant experiment with a human.

While a graduate student, Lindsley continued this work with adults with psychotic and schizophrenic disorders, as well as children with autism when he ran the Behavior Research Laboratory at Metropolitan State Hospital in Waltham, Massachusetts, for 12 years (Lindsley, 2001). During this time, he conducted applied research helping to change, for example, the residents' hallucinatory, vocalizing, and pacing behaviors to more socially appropriate behaviors. Using a manual plunger for reinforcement, patients could earn a penny, cigarette, M\&M, or a slice of apple, as a reinforcer. The lab closed in 1965 when 
Lindsley left to take a professorship in special education at the University of Kansas.

As early as May 1955, while still working with patients at the Metropolitan State Hospital, Lindsley began to use adaptations of semi-logarithmic graphs (Ogden R. Lindsley Archive). Realizing that the cumulative recorder would be far too cumbersome for the classroom, he began to develop the standard behavior chart with the aid of three of his graduate students-Eric Haughton, Ann Dell Duncan, and Carl Koenig-when he moved to Kansas.

It was typical fluid Ogden where he would run up the stairs with the latest version, one of us (Eric [sic], Carl [sic], myself) would put some data on it and run back down the stairs to see what he thought about the visualization. We went through several versions (3 cycle for example) until the six cycle emerged because it had to be something that could handle high frequency as well as low frequency behaviors. (A. D. Duncan, personal communication email to A. B. Calkin, January 26, 2020)

The chart developed rapidly, moving from Daily Graph 1 in March 1967 to Daily Chart 8 in December 1967.

When Eric Haughton graduated and then arrived at the University of Oregon for his first teaching position with his new $\mathrm{PhD}$, he brought the then-called daily graph, or DG-6, with him and he used it in his instruction. Behavior Research Company printed these graphs in September 1967. Haughton's first class included Calkin plus eight others, none of whom continued to chart after the class, with the exception of Calkin. Because of the rapid development of the daily chart (Daily Graph 1 to Daily Chart 8), no one kept data on the dates of development between the DG-1 and DC-8. The name for the initial chart was daily graph (DG), which became the standard behavior chart (SBC) used from December 1967 until at least 1972 (Pennypacker et al., 1972).

The chart, now referred to as the standard celeration chart (SCC), allows observers to look at any chart without having to pay special attention to the ordinate or abscissa axes, or as Lindsley referred to them, the "up (or down) and across" to know what the scale is. The "up and down" on the left is always the logarithmic scale, and the across is always a linear scale of time. This is why the standard exists. If the viewers know they are looking at a daily chart, there are always 140 days (or 20 weeks) on the "across." The weekly chart has 20 weeks. The monthly chart displays 1 decade, and the yearly chart 1 century. In each, the celeration line is $\mathrm{x} 2$ corner to corner (Caldwell, 1966; Calkin, 2005). Just as in medicine with the standard blood pressure, EKG, EEG, MRI, and C-T scan measures, looking at behavior needs to be standard also.
Thus, the family of SCCs has standard measurement at its core.

The techniques for using the SCC are well-described in The Handbook of the Standard Celeration Chart (Pennypacker et al., 2003). What are the benefits of the SCC? The benefits include its use of frequency, which is the basis for calculating and displaying both celeration and bounce, and its standard display. This enables the charter to view multiplicative change, including celeration, or proportional growth, and the variability of behavior. If one looks at a linear graph, which is additive in its display, the option of viewing the growth and natural variability of behavior and any outliers is not readily apparent. Further, behavior - whether cell division, fetal growth, disease, etc.- - changes by multiplying in any living organism. Even death shows a deceleration in the frequency of behavior. It is far easier to see and interpret the frequency of behaviors and graphically than using a table of numbers. One cannot look at growth or change additively any more than a shuttle can land on the moon or another planet when scientists view miles and time separately. All behavior change needs to be viewed and measured with the same precision as landing on the Moon or Mars. If its trajectory is a portion off, a space shuttle misses its target at great cost. Likewise, not tracking the trajectory of behavior and behavior change can cost an individual or any society a great deal of time, money, and effort in because we miss the target when attempting to create high quality education, health, learning, and standard of living. Just as someone steeped in physical science needs to look at the speed per unit of time of travel in space, those stepped in behavioral science need to look at the trajectory and patterns of frequency, celeration, and bounce of behavior across an individual's life. Those who use the SCC know they can predict a behavior's future with fine and detailed accuracy.

The behaviors pinpointed in the first charts created in the mid-1960s included inner behavior (Duncan, 1971; Sokolove, 1973), personal management behaviors (Green and Morrow, 1974), and learning in special education classes for emotionally disturbed students (Koenig, 1967). Because the field of education grew faster than the other two areas, the entire SCC measurement field using the SCC took on the name of precision teaching. Precision teaching, however, is but one application of the chart. Lindsley and others had originally hoped for precision inners, precision management, precision teaching, precision nursing, precision social work, etc. Unfortunately, however, the name "precision teaching" has stuck for all forms of the SCC. "Precision teaching" has been applied to many other areas since 1970, including business, medicine-including the tracking of international AIDS cases, and COVID-19 (Corso et al., 2021) - the military (Corso et al., 2017), law enforcement, sports, health fitness and wellness, and, again nonhuman animals, with the occasional training of dogs, chickens, wolves, and turtles. Although people (and nonhuman animals) are always learning 
and this learning is measured and can be charted on the SCC, it is problematic to continue to call the entire field "precision teaching." As Amy Evans and her colleagues state in their article in this issue, precision teaching is "a system for precisely defining and continuously measuring dimensional features of behavior and analyzing behavioral data . . . to make timely and effective data-based decisions to accelerate behavioral repertoires". But the science is not limited to precision teaching, and standard celeration charting encompasses all fields of human behavior, from fetal movement to reading to government to COVID-19 infections to the dying process.

This special section of Behavior Analysis in Practice focused on precision teaching offers readers a wide range of applications of the SCC, including reviews of the literature, conceptual articles, technical/tutorial articles, and empirical investigations. Amy Evans, Andrew Bulla, and Andrew Kieta provide an overview of the history of precision teaching and offers an updated definition of precision teaching in an effort to further differentiate precision teaching from other applications of standard celeration charting. The authors sought to address the misnomer that precision teaching functions as a type of instruction that makes learners do things quickly. Rather, the authors suggest precision teaching is a measurement system that allows teachers to make better and more timely decisions. Richard Kubina furthers this commentary on precision teaching, and highlights precision teaching's unique inductive research methods and analysis. Kubina provides some context for what readers can expect in the special section.

Kerri Milyko and Staheli Meyer and colleauges provide two technical articles offering step-by-step tutorials for advancements that precision teachers have made over the past few years. Milyko discusses how to apply k-schedule, a percentile schedule of reinforcement in which reinforcement is based on the current repertoire of the learner, to timings so one can shape behavioral frequencies within timed-practice sessions. This provides clinicians a practical way to train staff to deliver reinforcers for successive increases in behaviors targeted for increase. Meyer and colleagues provides an overview of the concept of agility and how one can use this conceptualization to analyze learning across time. They provide specific measures of agility and examples of what each measure demonstrates.

James Stocker and Richard Kubina, Mary Sawyer and colleagues, and Loraine Zanatta and Jesus Rosales-Ruiz provide readers some current investigations of precision teaching with general education populations. Stocker and Kubina contribute to the self-study literature, demonstrating an effective application of precision teaching and the SCC to build behavioral fluency in complex computation. This work extends the precision teaching math research to skills beyond more commonly targeted simple computation component skills.
Sawyer and colleagues detail their implementation of the Fit Lite $^{\mathrm{TM}}$ model, an instructional program derived from Fit Learning ${ }^{\mathrm{TM}}$, in a public school and demonstrates the associated effects of the program to their learners. In particular, the authors targeted specific components skills with fluency-based instruction and assessed the effects on a variety of curriculum-based measures. Lastly, Zanatta and Rosales-Ruiz presents an empirical investigation of the effects of different learning channel sets for the same skill area and the associated effects on acquisition, generalization, and retention. This article may provide precision teachers some additional considerations when selecting learning channel sets for their pinpoints for a wide array of academic skills.

The special section also offers some guidance on precision teaching applied to instructional strategies with individuals with autism and developmental disabilities. Sridhar Aravamudhan and Smita Awasthi and Luca Vascelli and colleagues used component-composite analyses to improve functional skills. Aravamudhan and Awasthi targeted component syllable sounds using frequency-building techniques and found improvements on composite-level words without direct intervention on those words. Likewise, Vascelli and colleagues targeted tool motor skills using frequency-building techniques and assessed their interaction with a variety of daily living skills. It should be noted that Vascelli and colleagues was erroneously published in a previous volume of Behavior Analysis in Practice, but was meant to be included in the special section. We encourage readers to go back and read the authors' work, because it contributes greatly to the precision teaching literature base. Both articles present a nice overview of such analyses, the utility of such analyses, and the power of building the frequencies of component-level skills. Lee Mason and Alonzo Andrews provide a pragmatic description of how to use precision-teaching methods within language-based interventions. The authors describe methods to probe the frequencies of verbal operants in a free-operant paradigm immediately after instruction. Lastly, Christina Barosky and colleagues focused on applying techniques derived from precision teaching to increase staff members' frequency of trials presented during teaching sessions. This article gives clinicians a practical approach to increase the pace in which their staff members deliver instructions during a teaching session.

One technique commonly used in behavior analysis that is derived from precision teaching is Say-All-Fast-Minute-EveryDay-Shuffled (SAFMEDS). The current special section presents two articles that further our empirical understanding of this technique. Shawn Quigley and colleagues assessed the effects of different procedural variations of SAFMEDS in an attempt to identify variables responsible for the greatest learning outcomes. Elizabeth Lovitz and colleagues present readers with a much-needed extension of SAFMEDS procedures for 
the digital age. The authors assessed a preliminary application of digital SAFMEDS in which they required learners to type in their responses rather than vocally state them. These articles present additional resources to individuals who use SAFMEDS as part of their training and/or teaching toolbox. In addition, Lovitz and colleagues provides guidance for professors instructing in an online format, which is particularly timely given the large shift to online instruction as a result of the global pandemic.

Finally, the special section includes three unique applications of precision teaching and standard celeration charting. First, Richard Kubina, Salvador Ruiz, and Douglas Kostewicz present a method for using the standard celeration chart to assist with experimental functional analyses as part of the functional behavior assessment (FBA) process. They propose a new version of the chart, the functional analysis celeration chart (FACC) and present archival data using the new chart to demonstrate its utility within the FBA process. Hafsa Junaid and colleagues targeted the physical activity of sedentary college students and used the SCC as part of a self-management package. Although not an instance of precision teaching, it shows how one can use the chart to promote physical activity and make decisions about the effectiveness of physical activity interventions. Lastly, Marisela Pallares and colleagues used precision teaching to teach tap dance movements to individuals. This highlights an interesting extension of precision teaching beyond general and special education settings and focuses on training nonacademic skills.

Taken together, these articles demonstrate that the field of precision teaching continues to grow and expand and that the SCC has a wide range of utility within the field of behavior analysis. We hope that these articles will spark new ideas and discoveries within the field.

\section{References}

Babe in a Box. (1952, May 2). Newsweek, 97-98. https://celeration.org/ wpcontent/uploads/2020/05/2006_JPTC_V22.01_04.pdf

Caldwell, T. E. (1966). Comparison of classroom measures: Percent, number, and rate. [Unpublished manuscript] Department of Special Education, University of Kansas Medical Center, Kansas City, Kansas.

Calkin, A. B. (2005). Precision teaching: The standard celeration charts. Behavior Analyst Today, 6, 207-215.

Corso, K. A., Meador, J., Kondis, M., \& Calkin, A. B. (2017). Evaluating system-level variables using applied behavior analysis. Report of NCR Behavioral Health, LLC, for the U.S. Air National Guard.

Corso, K. A., Kielbasa, K., \& Calkin, A. B. (2021). Using standard celeration makes COVID-19 data more meaningful. Families, Systems, \& Health, 39(1), 91-101.

Duncan, A. D. (1971). The view from the inner eye: Personal management of inner and outer behaviors. Teaching Exceptional Children, $3,152-156$.

Green, J., \& Morrow, W. (1974). Precision social work. In E. Thomas (Ed.), Behavior modification procedure: A sourcebook. Aldine Publishing Company

Koenig, C. H. (1967). Precision teaching with emotionally disturbed children. [Unpublished master's thesis] University of Kansas Medical Center, Kansas City, KS.

Lindsley, O. R. (2001). Studies in behavior therapy and behavior research therapy, June 1953-1965. In W. T. O’Donohue, D. A. Henderson, S. C. Hayes, J. E. Fisher, \& L. J. Hayes (Eds.), A history of the behavioral therapies: Founders' personal histories (pp. 125-153). Context Press.

Pennypacker, H. S., Koenig, C. H., \& Lindsley, O. R. (1972). Handbook of the standard behavior chart. Precision Media.

Pennypacker, H. S., Gutierrez, A., \& Lindsley, O. R. (2003). Handbook of the standard celeration chart. Cambridge Center for Behavioral Studies.

Sokolove, H. E. (1973). Inner behavior: The private eye becomes public information. Precision Media.

Publisher's Note Springer Nature remains neutral with regard to jurisdictional claims in published maps and institutional affiliations. 